\title{
Hybrid Orbitals and the Runge-Lenz Vector
}

\author{
LAWRENCE L. LOHR, JR. \\ Department of Chemistry, University of Michigan, \\ Ann Arbor, Michigan 48109, USA
}

\begin{abstract}
s
Matrix elements of the Runge-Lenz vector $\mathbf{A}$ are presented for those linear combinations of degenerate hydrogenic functions often referred to as hybrid orbitals. The uncertainties in the components of $\mathbf{A}$ for each type of wave-function are related to the distribution of classical Kepler orbits corresponding to each function. Matrix elements of $\mathbf{A}$ with respect to radially nodeless Slater functions are presented, as these functions are often used as a basis set in atomic and molecular calculations. The properties of $\mathbf{A}$ for a piecewise Coulombic central field are discussed in relation to the description of penetrating orbits in the old quantum theory. Simultaneous eigenfunctions of $\mathbf{A}$ and the Hamiltonian cannot be chosen for the piecewise Coulombic field because of a discontinuity in the radial derivative of the potential energy.
\end{abstract}

On présente des élements de la matrice représentant le vecteur de Runge-Lenz A dans une base consistant d'orbitales hybridées de fonctions hydrogénoïdes. Les mesures d'indétermination des composantes de $\mathbf{A}$ pour chaque type de fonction d'onde sont associées à la distribution des orbites de Kepler classiques correspondant à chaque fonction. On présente aussi des élements de la matrice de $\mathbf{A}$ par rapport à des fonctions de Slater sans nœuds. Les propriétés de A pour un champ Coulombien central par morceaux sont discutées par rapport à la description des orbites pénétrantes dans la vieille théorie quantique. Il n'est pas possible de choisir des fonctions propres simultanées de $\mathbf{A}$ et du Hamiltonien, à cause d'une discontinuité dans la dérivée radiale de l'énergie potentielle.

Matrixelemente des Runge-Lenz'schen Vektors A mit Rücksicht auf von entarteten Wasserstoffunktionen konstruierte Hybridorbitale werden angegeben. Die Unbestimmtheiten in den Komponenten von $\mathbf{A}$ für jeden Typ von Wellenfunktionen sind mit der jeder Funktion entsprechenden Verteilung der klassischen Keplerbahnen verbunden. Matrixelemente von $\mathbf{A}$ werden auch mit Rücksicht auf knotenlose, radielle Slaterfunktionen angegeben. Die Eigenschaften von $\mathbf{A}$ für ein stückweise Coulombisches Zentralfeld werden in Bezug auf die Beschreibung von penetrierenden Bahnen in der alten Quantentheorie diskutiert. Gleichzeitige Eigenfunktionen von $\mathbf{A}$ und vom Hamiltonoperator können für das stückweise Coulombische Feld wegen einer Diskontinuität in der radiellen Ableitung der potentiellen Energie nicht gewählt werden.

It has been known for some time that the "accidental" degeneracy associated with the nonrelativistic description of the bound states of hydrogenic atoms may be related $[1,2]$ to the invariance of the Hamiltonian with respect to the four-dimensional orthogonal symmetry group $\mathrm{O}_{4}$. Alternatively, the degeneracy may be related $[1,3]$ to the existence of a vector constant of motion in addition to the orbital angular momentum vector. This vector is called [4] the Runge-Lenz vector $\mathbf{A}$ and may be written $[1-3]^{*}$ as

$$
\mathbf{A}=1 / 2\{(\mathbf{p} \times \mathbf{L})-(\mathbf{L} \times \mathbf{p})\}-P \mathbf{r} / r
$$

${ }^{*}$ Several definitions of $\mathbf{A}$ exist which differ by multiplicative factors. We follow Englefield [2], whose $\mathbf{A}$ has dimensions of momentum. The $\mathbf{A}$ of Bander and Itzykson [1] has the same sign but dimensions of charge squared, while that of Pauli [3] is of opposite sign and dimensionless.

(C) 1976 by John Wiley \& Sons, Inc. 
where $\mathbf{p}$ is the linear momentum operator, $\hbar \mathbf{L}$ is the orbital angular momentum operator ( $\hbar$ is Planck's constant divided by $2 \pi$ ), $\mathbf{r}$ is the electron radius vector, and $P$ is a constant $\mu Z e^{2} / \hbar$ with the dimensions of momentum where $\mu$ is the reduced mass, $Z$ is the atomic number, and $e$ is the magnitude of electron charge. The constant $P$ may also be written as $Z \hbar / a_{\mathrm{H}}$, in which $a_{\mathrm{H}}=\hbar^{2} / \mu e^{2}=(m / \mu) a_{0}$ where $m$ is the electron mass and $a_{0}$ is the Bohr radius for infinite nuclear mass. Classically, the vector $\mathbf{A}$ lies along the major axis of an elliptical orbit and has a magnitude equal to $P$ times the eccentricity of the orbit. For the sign convention in Eq. (1), $\mathbf{A}$ points in the direction of the perihelion of the orbit.

The Runge-Lenz vector has attracted considerable attention in recent years because of its usefulness in approximately describing [5-12] the dynamical symmetry in two-electron atoms. Specifically, configuration mixing coefficients for doubly excited states have been obtained [8-12] group-theoretically by considering a basis which is diagonal in the square of the difference of the Runge-Lenz vectors of the electrons and subject to conservation of parity and of orbital and spin angular momenta. Other applications have included the evaluation [13] of Coulomb integrals with respect to hydrogenic wave-functions and the determination [14] of a "hidden" constant of motion for the two-center Coulomb problem. Some developments in the analysis of the dynamical symmetries of classical systems included the demonstration $[15,16]$ of the existence of a RungeLenz-type vector for all spherically symmetric single-particle potentials in three dimensions.

The basic commutation relationships involving $\mathbf{A}, \mathbf{L}$, and the hydrogenic Hamiltonian $\mathscr{H}$ have been given by several authors [1-3] and are

$$
\begin{aligned}
{\left[A_{j}, A_{k}\right] } & =-2 i \epsilon_{j k l} \mu L_{l} \mathscr{H} \\
{\left[L_{j}, A_{k}\right] } & =i \epsilon_{j k l} A_{l} \\
{\left[L_{j}, L_{l}\right] } & =i \epsilon_{j k l} L_{l} \\
{\left[\mathscr{H}, A_{j}\right] } & =\left[\mathscr{H}, L_{j}\right]=0
\end{aligned}
$$

$$
\mathbf{A} \cdot \mathbf{L}=\mathbf{L} \cdot \mathbf{A}=0
$$

where the factor $\epsilon_{j k l}$ is the Levi-Civita tensor.*

The vector $\mathbf{A}$ has been used to derive matrix elements of the position vector $\mathbf{r}$ without explicitly using radial wave-functions. The key result, obtained [2] by Englefield using a hypervirial relationship, is

$$
4 \mu E_{n}\left\langle n l \| \mathbf{r} \mid n l^{\prime}\right\rangle=3 \hbar\left\langle n l\|\mathbf{A}\| n l^{\prime}\right\rangle
$$

in which the energy eigenvalues $E_{n}$ are

$$
E_{n}=\frac{-\mu Z^{2} e^{4}}{2 \hbar^{2} n^{2}}=\frac{-P^{2}}{2 \mu n^{2}}
$$

\footnotetext{
* The tensor $\epsilon_{j k l}$ equals zero if any two subscripts are equal, equals +1 if $j k l$ represents an even permutation of $x y z$, and equals -1 if $j k l$ represents an odd permutation of $x y z$.
} 
The double bars in Eq. (3) denote reduced matrix elements related to matrix elements by the Wigner-Eckart theorem, which for complex components may be written

$$
\left\langle n l m\left|l^{\prime \prime} m^{\prime \prime}\right| n l^{\prime} m^{\prime}\right\rangle=(-1)^{l-m}\left\langle n l\left\|l^{\prime \prime}\right\| n l^{\prime}\right\rangle \bar{V}\left(\begin{array}{ccc}
l & l^{\prime} & l^{\prime \prime} \\
-m & m^{\prime} & m^{\prime \prime}
\end{array}\right)
$$

where $\bar{V}$ is the vector coupling coefficient of Fano and Racah [17]. Alternatively, the use of Griffith's $V$ coefficients [18] for real components yields

$$
\left\langle\alpha \Gamma M_{\Gamma}\left|\Gamma^{\prime \prime} M_{\Gamma}^{\prime \prime}\right| \alpha^{\prime} \Gamma^{\prime} M_{\Gamma}^{\prime}\right\rangle=\left\langle\alpha \Gamma\left\|\Gamma^{\prime \prime}\right\| \alpha^{\prime} \Gamma^{\prime}\right\rangle V\left(\begin{array}{ccc}
\Gamma & \Gamma^{\prime} & \Gamma^{\prime \prime} \\
M_{\Gamma} & M_{\Gamma}^{\prime} & M_{\Gamma}^{\prime \prime}
\end{array}\right)
$$

in which the quantum numbers $\alpha, \Gamma$, and $M_{\Gamma}$ are analogous to $n, l$, and $m$, respectively.

Since $\mathbf{A}$ commutes with the hydrogenic Hamiltonian, the matrix of $\mathbf{A}$ is diagonal in the principal quantum number $n$, and Eq. (3) cannot be used to obtain matrix elements of $\mathbf{r}$ between states of different $n$. The fact that matrix elements of A between states of the same $n$ are simply proportional to those of $\mathbf{r}$ might make it seem that there is little use in discussing the former except as a route to the latter. However, as we shall show, it is the special property of $\mathbf{A}$ being diagonal in $n$ which makes it useful for an analysis of the properties of hydrogenic hybrid orbitals.

Pauli showed [3] that the eigenstates for a hydrogenic atom in a weak electric field may be chosen as simultaneous eigenstates of the components of $\mathbf{A}$ and $\mathbf{L}$ parallel to the field. For $n=2$ these states are the pair of functions often described as digonal $s p$ hybrids together with the pair of $p$ functions with $M_{l}= \pm 1$. Choosing $z$ as the field direction, these four functions are eigenstates of $A_{z}$, but not of $A_{x}$ and $A_{y}$, as the latter operators mix the digonal hybrids with $2 p_{x}$ and $2 p_{y}$ functions, respectively. The operation of $A_{z}$ on the familiar real $n=2$ functions gives

$$
\begin{aligned}
A_{z}|2 s\rangle & =(P / 2)\left|2 p_{z}\right\rangle \\
A_{z}\left|2 p_{x}\right\rangle & =0 \\
A_{z}\left|2 p_{y}\right\rangle & =0 \\
A_{z}\left|2 p_{z}\right\rangle & =(P / 2)|2 s\rangle
\end{aligned}
$$

with similar results for $A_{x}$ and $A_{y}$. Thus, $A_{z}$ simply interchanges the functions $2 s$ and $2 p_{z}$. The reduced matrix element ${ }^{*}\langle 2 s\|\mathbf{A}\| 2 p\rangle$ is $3^{1 / 2} P / 2$. As given by Pauli, but using Englefield's sign convention [2], the eigenvalues of $A_{z}$ are $\pm P / 2$ for the digonal hybrids $2^{-1 / 2}\left[|2 s\rangle \pm\left|2 p_{z}\right\rangle\right]$ and zero for $2 p_{+}$and $2 p_{-}$(or equivalently for $2 p_{x}$ and $2 p_{y}$ ). In addition, each digonal hybrid is connected to $2 p_{x}$ by a matrix

* The positive sign for $\left\langle n l\|\mathbf{A}\| n l^{\prime}\right\rangle$ corresponds, using Eq. (3), to a negative sign for $\left\langle n l\|\mathbf{r}\| n l^{\prime}\right\rangle$. This implies a choice of radial phases such that the lobe closest to the nucleus has the same sign, say positive, for all states. Thus the outer lobe of $|2 s\rangle$ is negative, making the density associated with the digonal hybrid $2^{-1 / 2}\left[|2 s\rangle+\left|2 p_{z}\right\rangle\right]$ point along the negative $z$ axis. 
element of $A_{x}$ equaling $8^{-1 / 2} P$ and to $2 p_{y}$ by a matrix element of $A_{v}$ equaling $8^{-1 / 2} P$.

A trigonal set of $s p^{2}$ functions for $n=2$ may be defined by

$$
\begin{aligned}
& \psi_{1}=3^{-1 / 2}\left[|2 s\rangle+2^{1 / 2}\left|2 p_{x}\right\rangle\right] \\
& \psi_{2}=3^{-1 / 2}\left[|2 s\rangle-2^{-1 / 2}\left|2 p_{x}\right\rangle+(3 / 2)^{1 / 2}\left|2 p_{y}\right\rangle\right] \\
& \psi_{3}=3^{-1 / 2}\left[|2 s\rangle-2^{-1 / 2}\left|2 p_{x}\right\rangle-(3 / 2)^{1 / 2}\left|2 p_{y}\right\rangle\right]
\end{aligned}
$$

In Table I we present the matrices of $A_{x}, A_{y}$, and $A_{z}$ with respect to the functions in Eq. (8) together with $\psi_{4}=\left|2 p_{z}\right\rangle$. The diagonal elements are the direction cosines of Eq. (8) multiplied by the constant $2^{1 / 2} P / 3$ and are components of the vector

\begin{tabular}{|c|c|c|c|c|}
\hline & $\psi_{1}$ & $\psi_{2}$ & $\psi_{3}$ & $\psi_{4}$ \\
\hline \multirow{4}{*}{$A_{x}$} & $\psi_{1}(8 / 3)^{1 / 2}$ & $6^{-1 / 2}$ & $6^{-1 / 2}$ & 0 \\
\hline & $\psi_{2} \quad 6^{-1 / 2}$ & $-(2 / 3)^{1 / 2}$ & $-(2 / 3)^{1 / 2}$ & 0 \\
\hline & $6^{-1 / 2}$ & $-(2 / 3)^{1 / 2}$ & $-(2 / 3)^{1 / 2}$ & 0 \\
\hline & $\psi_{4}$ & 0 & 0 & 0 \\
\hline \multirow{4}{*}{$A_{y}$} & $\psi_{1}$ & $2^{-1 / 2}$ & $-2^{-1 / 2}$ & 0 \\
\hline & $\psi_{2} \quad 2^{-1 / 2}$ & $2^{1 / 2}$ & 0 & 0 \\
\hline & $\psi_{3} \quad-2^{-1 / 2}$ & 0 & $-2^{1 / 2}$ & 0 \\
\hline & $\psi_{4}$ & 0 & 0 & 0 \\
\hline \multirow{4}{*}{$A_{z}$} & $\psi_{1}$ & 0 & 0 & 1 \\
\hline & $\psi_{2}$ & 0 & 0 & 1 \\
\hline & $\psi_{3}$ & 0 & 0 & 1 \\
\hline & $\psi_{4}$ & 1 & 1 & 0 \\
\hline
\end{tabular}
expectation value

TABLE I. Matrix elements ${ }^{a}$ of $\mathbf{A}$ in $s p^{2}$ basis $^{b}$ for $n=2$.

${ }^{a}$ In units of $12^{-1 / 2} P$.

${ }^{\mathrm{b}}$ Basis defined by Eq. (8) together with $\psi_{4}=\left|2 p_{z}\right\rangle$.

$$
\langle\psi|\mathbf{A}| \psi\rangle=\left\langle\psi \mid \boldsymbol{A}_{x} \| \psi\right\rangle \mathbf{i}+\left\langle\psi\left|\boldsymbol{A}_{y}\right| \psi\right\rangle \mathbf{j}+\left\langle\psi\left|\boldsymbol{A}_{z}\right| \psi\right\rangle \mathbf{k}
$$

where $\mathbf{i}, \mathbf{j}$, and $\mathbf{k}$ are unit vectors in the $x, y$, and $z$ directions, respectively. Thus, as indicated by Eq. (3), the expectation value of $\mathbf{A}$ lies antiparallel to the mean position of the charge density. The nonvanishing of Eq. (9) for the pair of digonal functions and for $\psi_{1}, \psi_{2}$, and $\psi_{3}$ indicates that each of these hybrid functions corresponds to a set of classical elliptical orbits whose mean $\mathbf{A}$ value equals the expectation value.

Similar properties hold for the set of four $n=2 s p^{2}$ hybrid functions

$$
\begin{aligned}
& \psi_{a}=1 / 2\left[|2 s\rangle+\left|2 p_{x}\right\rangle+\left|2 p_{y}\right\rangle+\left|2 p_{z}\right\rangle\right] \\
& \psi_{b}=1 / 2\left[|2 s\rangle-\left|2 p_{x}\right\rangle-\left|2 p_{y}\right\rangle+\left|2 p_{z}\right\rangle\right] \\
& \psi_{c}=1 / 2\left[|2 s\rangle-\left|2 p_{x}\right\rangle+\left|2 p_{y}\right\rangle-\left|2 p_{z}\right\rangle\right] \\
& \psi_{d}=1 / 2\left[|2 s\rangle+\left|2 p_{x}\right\rangle-\left|2 p_{y}\right\rangle-\left|2 p_{z}\right\rangle\right]
\end{aligned}
$$

for which we present the matrix elements of $A_{x}, A_{y}$, and $A_{z}$ in Table II. 
TABLE II. Matrix elements ${ }^{a}$ of $\mathbf{A}$ in $s p^{3}$ basis $^{b}$ for $n=2$.

\begin{tabular}{cccrrr}
\hline & & $\psi_{a}$ & $\psi_{b}$ & $\psi_{c}$ & $\psi_{d}$ \\
\hline \multirow{4}{*}{$A_{x}$} & $\psi_{a}$ & 1 & 0 & 0 & 1 \\
& $\psi_{b}$ & 0 & -1 & -1 & 0 \\
& $\psi_{c}$ & 0 & -1 & -1 & 0 \\
& $\psi_{d}$ & 1 & 0 & 0 & 1 \\
& $\psi_{a}$ & 1 & 0 & 1 & 0 \\
$A_{y}$ & $\psi_{b}$ & 0 & -1 & 0 & -1 \\
& $\psi_{c}$ & 1 & 0 & 1 & 0 \\
& $\psi_{d}$ & 0 & -1 & 0 & -1 \\
& $\psi_{a}$ & 1 & 1 & 0 & 0 \\
$A_{z}$ & $\psi_{b}$ & 1 & 1 & 0 & 0 \\
& $\psi_{c}$ & 0 & 0 & -1 & -1 \\
& $\psi_{d}$ & 0 & 0 & -1 & -1 \\
\hline$=$
\end{tabular}

Basis defined by Eq. (10).

' In units of $P / 4$.

The spread $\sigma$ associated with the $i$ th component of $\mathbf{A}$ is given by

$$
\sigma^{2}\left(A_{i}\right)=\left\langle\psi\left|A_{i}^{2}\right| \psi\right\rangle-\left|\left\langle\psi\left|A_{i}\right| \psi\right\rangle\right|^{2}
$$

For the $s p^{2}$ hybrids in Eq. (8),

$$
\begin{aligned}
& \left\langle\psi_{1}\left|A_{x}^{2}\right| \psi_{1}\right\rangle=P^{2} / 4 \\
& \left\langle\psi_{2}\left|A_{x}^{2}\right| \psi_{2}\right\rangle=\left\langle\psi_{3}\left|A_{x}^{2}\right| \psi_{3}\right\rangle=P^{2} / 8 \\
& \left\langle\psi_{1}\left|A_{y}^{2}\right| \psi_{1}\right\rangle=P^{2} / 12 \\
& \left\langle\psi_{2}\left|A_{y}^{2}\right| \psi_{2}\right\rangle=\left\langle\psi_{3}\left|A_{y}^{2}\right| \psi_{3}\right\rangle=P^{2} / 6 \\
& \left\langle\psi_{i}\left|A_{z}^{2}\right| \psi_{i}\right\rangle=P^{2} / 12(\text { for } i=1-3)
\end{aligned}
$$

Combining Eq. (12) with matrix elements of $\mathbf{A}$ in Table I yields: $\sigma\left(A_{x}\right)$ values of $P / 6$ for $\psi_{1}$ and $(5 / 72)^{1 / 2} P$ for $\psi_{2}$ and $\psi_{3} ; \sigma\left(A_{y}\right)$ values of $12^{-1 / 2} P$ for $\psi_{1}$ and $6^{-1 / 2} P$ for $\psi_{2}$ and $\psi_{3}$; and $\sigma\left(A_{z}\right)$ values of $12^{-1 / 2} P$ for $\psi_{i}$, where $i=1-3$. The smallest of these values is $\sigma\left(A_{x}\right)$ for $\psi_{1}$, indicating simply that the orientation of the function in Eq. (8) with respect to the Cartesian axes places $\psi_{1}$ along the $x$ axis. The functions $\psi_{1}, \psi_{2}$, and $\psi_{3}$ are equivalent by symmetry and must have identical properties. However, it is significant that $\sigma\left(A_{y}\right)$ and $\sigma\left(A_{z}\right)$ for $\psi_{1}$ are each larger by a factor of $3^{1 / 2}$ than $\sigma\left(A_{x}\right)$ for $\psi_{1}$, indicating that $\psi_{1}$ is closer to being an eigenfunction of $A_{x}$ than of $A_{y}$ or $A_{z}$. Since it can easily be shown from the "interchange" properties in Eq. (7) that any superposition of $s$ and $p$ functions for $n=2$ is an eigenfunction of the square of the component of $\mathbf{A}$ parallel to the direction of the mean charge density, it follows that $\psi_{1}$ is an eigenfunction of $A_{x}^{2}$, although not of $A_{x}$. 
By contrast, the $s p$ hybrids, which are eigenfunctions of $A_{z}$, are characterized by $\sigma\left(A_{z}\right)=0$ and $\sigma\left(A_{x}\right)=\sigma\left(A_{y}\right)=8^{-1 / 2} P$. The latter follows from the fact that the mean values of $A_{x}^{2}$ and $A_{y}^{2}$ are $P^{2} / 8$ while the mean values of $A_{x}$ and $A_{y}$ are zero. Thus, the hybrids $2^{-1 / 2}\left[|2 s\rangle \pm\left|2 p_{z}\right\rangle\right]$ correspond to sets of elliptical orbits having $A_{z}= \pm P / 2$, respectively, but with a spread of $A_{x}$ and $A_{y}$ values. Specifically, the magnitude of $A_{z}$ for these hybrids is $2^{-1 / 2}$ times that of $\mathbf{A}$ itself, as it is for an elliptical orbit of any orientation that places the major axis at an angle of $45^{\circ}$ to the $z$ axis. If, in addition, the plane of the orbit contains the $z$ axis, then $L_{z}$ is zero and the correspondence is even closer.

For each of the four $s p^{2}$ hybrid $n=2$ functions in Eq. (10)

$$
\left\langle\psi\left|A_{x}^{2}\right| \psi\right\rangle=\left\langle\psi\left|A_{y}^{2}\right| \psi\right\rangle=\left\langle\psi\left|A_{z}^{2}\right| \psi\right\rangle=P^{2} / 8
$$

and

$$
\left|\left\langle\psi\left|A_{x}\right| \psi\right\rangle\right|=\left|\left\langle\psi\left|A_{y}\right| \psi\right\rangle\right|=\left|\left\langle\psi\left|A_{z}\right| \psi\right\rangle\right|=P / 4
$$

so that

$$
\sigma\left(A_{x}\right)=\sigma\left(A_{y}\right)=\sigma\left(A_{z}\right)=P / 4
$$

meaning that the spread in each component of $\mathbf{A}$ equals the magnitude of the mean value of that component.

The properties of states with $n>2$ may be illustrated by the operation of $\mathbf{A}$ on the familiar real eigenstates of $L^{2}$ for $n=3$ :

$$
A_{x}\left|3 p_{y}\right\rangle=A_{y}\left|3 p_{x}\right\rangle=3^{-1} P\left|3 d_{x y}\right\rangle
$$

with similar relationships involving $x, z$ and $y, z$ :

$$
\begin{gathered}
A_{x}\left|3 p_{x}\right\rangle=(8 / 27)^{1 / 2} P|3 s\rangle-27^{-1 / 2} P\left|3 d_{3 z^{2}-r^{2}}\right\rangle \\
+3^{-1} P\left|3 d_{x^{2}-y^{2}}\right\rangle
\end{gathered}
$$

with a change in sign of the last term for $A_{y}\left|3 p_{y}\right\rangle$; and

$$
A_{z}\left|3 p_{z}\right\rangle=(8 / 27)^{1 / 2} P|3 s\rangle+(4 / 3)^{1 / 2} P\left|3 d_{3 z^{2}-r^{2}}\right\rangle
$$

The results are also presented as the matrix elements in Table III. Reduced matrix elements for use in Eq. (6) with coupling coefficients for the $O_{h}$ group are

$$
\begin{gathered}
\left\langle 3 s\left(a_{1 g}\right)\left\|\mathbf{A}\left(t_{1 u}\right)\right\| 3 p\left(t_{1 u}\right)\right\rangle=8^{1 / 2} P / 3 \\
\left\langle 3 p\left(t_{1 u}\right)\left\|\mathbf{A}\left(t_{1 u}\right)\right\| 3 d\left(e_{g}\right)\right\rangle=-2 P / 2 \\
\left\langle 3 p\left(t_{1 u}\right)\left\|\mathbf{A}\left(t_{1 u}\right)\right\| 3 d\left(t_{2 g}\right)\right\rangle=-6^{1 / 2} P / 3
\end{gathered}
$$

The simultaneous eigenfunctions of $A_{z}$ and $L_{z}$ are the spd linear hybrids with $M_{L}=0$, the $p d$ linear hybrids with $M_{L}= \pm 1$, and the unmixed $d$ functions with $M_{L}= \pm 2$. The properties of $\mathbf{A}$ with respect to hybrid $n=3$ functions are similar to those we have discussed for $n=2$, so that the spread in the component of $\mathbf{A}$ parallel to the direction of an $s p^{3} d^{2}$ hybrid is small compared to the spread in perpendicular components. 
TABLE III. Matrix elements ${ }^{\mathrm{a}}$ of $\mathbf{A}$ for $n=3$.

\begin{tabular}{|c|c|c|c|c|c|c|c|}
\hline & & $s$ & $d_{3 z^{2}-r^{2}}$ & $d_{x^{2}-y^{2}}$ & $d_{x y}$ & $d_{x z}$ & $d_{y z}$ \\
\hline \multicolumn{3}{|c|}{$p_{x}(8 / 3)^{1 / 2}$} & $-3^{-1 / 2}$ & 1 & 0 & 0 & 0 \\
\hline \multirow[t]{2}{*}{$A_{x}$} & $p_{y}$ & 0 & 0 & 0 & 1 & 0 & 0 \\
\hline & $p_{z}$ & 0 & 0 & 0 & 0 & 1 & 0 \\
\hline \multirow{3}{*}{$A_{y}$} & $p_{x}$ & 0 & 0 & 0 & 1 & 0 & 0 \\
\hline & $p_{y}$ & $(8 / 3)^{1 / 2}$ & $-3^{-1 / 2}$ & -1 & 0 & 0 & 0 \\
\hline & $p_{z}$ & 0 & 0 & 0 & 0 & 0 & 1 \\
\hline \multirow{3}{*}{$A_{z}$} & $p_{x}$ & 0 & 0 & 0 & 0 & 1 & 0 \\
\hline & $p_{y}$ & 0 & 0 & 0 & 0 & 0 & 1 \\
\hline & $p_{z}$ & $(8 / 3)^{1 / 2}$ & $(4 / 3)^{1 / 2}$ & 0 & 0 & 0 & 0 \\
\hline
\end{tabular}

${ }^{\text {a }}$ In units of $P / 3$.

The hybrid functions used to describe bonding in molecules are usually taken not as superpositions of hydrogenic functions but rather of atomic central-field functions. The matrix of $\mathbf{A}$ is not diagonal in the principal quantum number $n$ for such functions, which are frequently determined by a self-consistent field (SCF) procedure involving an expansion in either a Slater or Gaussian basis set. A Slater $s$ function may be written

$$
|n s\rangle=\left[\frac{(2 \alpha)^{2 n+1}}{4 \pi(2 n) !}\right]^{1 / 2} r^{n-1} e^{-\alpha r}=N_{s} r^{n-1} e^{-\alpha r}
$$

so that

$$
A_{z}|n s\rangle=N_{s}\left[(\hbar \alpha-P)-\frac{\hbar(n-1)}{r}\right] r^{n-1} e^{-\alpha r} \cos \theta
$$

Since a Slater $p_{z}$ function may be written

$$
\left|n p_{z}\right\rangle=\left[\frac{3(2 \beta)^{2 n+1}}{4 \pi(2 n) !}\right]^{1 / 2} r^{n-1} e^{-\beta r} \cos \theta=N_{p} r^{n-1} e^{-\beta r} \cos \theta
$$

we have

$$
\begin{aligned}
\left\langle n p_{z}\left|A_{z}\right| n^{\prime} s\right\rangle= & (4 \pi / 3) N_{s} N_{p} \frac{\left(n+n^{\prime}-1\right) !}{(\alpha+\beta)^{n+n^{\prime}+1}} \\
& \times\left[(\hbar \alpha-P)\left(n+n^{\prime}\right)-\hbar\left(n^{\prime}-1\right)(\alpha+\beta)\right]
\end{aligned}
$$

which is not diagonal in $n$. For $n^{\prime}=1$, the second term in Eqs. (19) and (21) vanishes, while the first term approaches zero as $\hbar \alpha$ approaches $P=\hbar Z / a_{\mathrm{H}}$, the hydrogenic value of $\hbar \alpha$ for $n^{\prime}=1$. Thus, as expected, $\mathbf{A}$ annihilates a hydrogenic $1 s$ function. For $n=n^{\prime}=2$ and $\alpha=\beta=Z / 2 a_{\mathrm{H}},\left\langle 2 p_{z}\left|A_{z}\right| 2 s\right\rangle$ from Eqs. (21) equals $-3^{1 / 2} P / 4$, as compared to the hydrogenic value of $P / 2$. The sign difference arises trivially from the fact that the phase of the Slater $|2 s\rangle$ function (18) is positive while that of the corresponding outer lobe of the hydrogenic $|2 s\rangle$ function is negative. 
The value of $-3^{1 / 2} P / 4$ is unchanged if the Slater $|2 s\rangle$ function is Schmidtorthogonalized to a Slater $|1 s\rangle$ function with the same exponent as the $|2 s\rangle$ function, namely $Z / 2 a_{\mathrm{H}}$. The resulting $|2 s\rangle$ function differs from the hydrogenic $|2 s\rangle$ function in having its radial node at $3 a_{\mathrm{H}} / Z$ instead of $2 a_{\mathrm{H}} / Z$ and by having a normalization constant larger by a factor of $12^{1 / 2} / 3$. While a diagonal hydrogenic hybrid $2^{-1 / 2}\left[|2 s\rangle+\left|2 p_{z}\right\rangle\right]$ is an eigenfunction of both $A_{z}$ and $A_{z}^{2}$, with $\sigma\left(A_{z}\right)=0$, the corresponding Slater hybrid constructed with either the radially nodeless or the orthogonalized $|2 s\rangle$ function is not an eigenfunction of either operator but has $\sigma\left(A_{z}\right)=5^{1 / 2} P / 12$, a value obtained from the matrix elements $\left\langle 2 s\left|A_{z}^{2}\right| 2 s\right\rangle=$ $7 P^{2} / 36,\left\langle 2 p_{z}\left|A_{z}^{2}\right| 2 p_{z}\right\rangle=P^{2} / 4$, and $\left\langle 2 p_{z}\left|A_{z}\right| 2 s\right\rangle=-3^{1 / 2} P / 4$. The expectation value of $A_{z}$ for the Slater hybrid is also $-3^{1 / 2} P / 4$, smaller in magnitude than the hydrogenic value of $P / 2$. While these results are for $s p$ hybrids using single Slater functions, Eq. (21) may be used to evaluate matrix elements of $\mathbf{A}$ with respect to any $s p^{n}$ hybrids constructed from atomic functions which are superpositions of any number of Slater basis functions.

To obtain matrix elements connecting Slater $p$ and $d$ functions we use

$$
A_{z}\left|n p_{z}\right\rangle=\left\{(\hbar k-P) / 3+\hbar / r-\left(3 \cos ^{2} \theta-1\right)(2 \hbar k+P) / 3\right\} N_{p} r_{n-1} e^{-\beta r}
$$

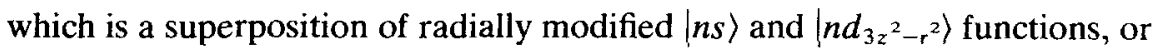

$$
A_{x}\left|n p_{z}\right\rangle=-\{2 \hbar k+P\} N_{p} r^{n-1} e^{-\beta r} \sin \theta \cos \theta \cos \phi
$$

which is a modified $\left|n d_{x y}\right\rangle$ function; the factor $k$ in Eqs. (22) and (23) equals $[(n-2) / r]-\beta$. The matrix element $\left\langle n^{\prime} s\left|A_{z}\right| n p_{z}\right\rangle$ is the same as that given in Eq. (21), while

$$
\left\langle n^{\prime} d_{x z}\left|A_{x}\right| n p_{z}\right\rangle=-(4 \pi / 15) N_{p} N_{d}\{2 \hbar k+P\} \frac{\left(n+n^{\prime}\right) !}{(\beta+\gamma)^{n+n^{\prime}+1}}
$$

where $\gamma$ is the exponent for $\left|n^{\prime} d_{x z}\right\rangle$ and $N_{d}$ is the normalization constant $\left[(15 / 4 \pi)(2 \gamma)^{2 n^{\prime}+1} /\left(2 n^{\prime} !\right)\right]^{1 / 2}$.

Other matrix elements involving $\left|n^{\prime} d\right\rangle$ and $|n p\rangle$ functions are readily obtained from Eq. (24) by the use of coupling coefficients. Reduced matrix elements thus obtained from Eqs. (21) or (24) can then be used to scale the matrix elements in Tables I-III to a non-hydrogenic basis.

An interesting example of a central field is the piecewise Coulombic field corresponding to a positive nucleus surrounded by spherical shells of negative charge. This field has been used by McGuire [19] and by Chapman and Lohr [20] in calculating continuum wave-functions and photoionization cross sections. Since the field is Coulombic in each region of space, hydrogenic wave-functions matched at each shell radius form exact solutions of the Schrödinger equation. However, the bound states do not possess the $n^{2}$ (excluding spin) hydrogenic degeneracy. It is instructive to examine carefully the commutator $[\mathbf{A}, \mathscr{H}]$ when $\mathscr{H}$ contains a piecewise Coulombic potential energy. We first write

$$
\mathbf{A}=\mathbf{A}_{1}+\mathbf{A}_{2}
$$


where

$$
\mathbf{A}_{1}=1 / 2[(\mathbf{p} \times \mathbf{L})-(\mathbf{L} \cdot \mathbf{p})]
$$

and

$$
\mathbf{A}_{2}=-P \mathbf{r} / r
$$

Thus,

$$
[\mathbf{A}, \mathscr{H}]=\left[\mathbf{A}_{1}, T\right]+\left[\mathbf{A}_{1}, V\right]+\left[\mathbf{A}_{2}, T\right]+\left[\mathbf{A}_{2}, V\right]
$$

Since $\mathbf{A}_{2}$ and $V$ are multiplicative operators, $\left[\mathbf{A}_{2}, V\right.$ ] equals zero. If $V$ is a function of $r$ only, then

$$
\left[\mathbf{A}_{1}, V\right]=-i(d V / d r)\left[r^{-1}(\mathbf{r} \cdot \mathbf{p}) \mathbf{r}-r \mathbf{p}\right]
$$

From Englefield [2],

$$
\left[\mathbf{A}_{2}, T\right]=(i \hbar P / 2 \boldsymbol{\mu})\left\{\boldsymbol{r}^{-3}[\mathbf{r} \cdot \mathbf{p}+\mathbf{p} \cdot \mathbf{r}+3 i \hbar] \mathbf{r}-2 r^{-1} \mathbf{p}\right\}
$$

For a hydrogenic system $d V / d r=Z e^{2} / r^{2}$, so that

$$
\left[\mathbf{A}_{1}, V\right]=-\left[\mathbf{A}_{2}, T\right]
$$

if $P$ in $\mathbf{A}_{2}$ is set equal to $Z \hbar / a_{\mathbf{H}}=Z e^{2} \mu / \hbar$. The remaining term in Eq. (26), $\left[\mathbf{A}_{1}, T\right]$, equals zero assuming* that it operates on functions which are analytic. Thus, from Eq. (26) the well known result is $[\mathbf{A}, \mathscr{H}]=0$. For smooth but non-Coulombic central fields Eq. (29) no longer holds, so that $[\mathbf{A}, \mathscr{H}] \neq 0$, meaning that $\mathbf{A}$ is no longer a constant of motion. However, for the piecewise Coulombic field for which $V(r)=-\left(Z_{i}^{\text {eff }} e^{2} / r\right)+C_{i}$ in the $i$ th region, where $Z_{i}^{\text {eff }} e$ is the effective nuclear charge and $C_{i}$ is the external shielding constant, the commutator $\left[\mathbf{A}_{1}, V\right]$ again equals $-\left[\mathbf{A}_{2}, T\right]$, as in Eq. (29), if $P$ is set equal to $Z_{i}^{\text {eff }} \hbar / a_{\mathrm{H}}$. The reason is that both commutators are proportional to $Z_{i}^{\text {eff }}$, so that even though $d V / d r$ in $\left[\mathbf{A}_{1}, V\right]$ is discontinuous, the change is matched by the change in $P$ in $\left[\mathbf{A}_{2}, T\right]$. We know that $[\mathbf{A}, \mathscr{H}]$ cannot be zero for the piecewise Coulombic field, so we must examine the behavior of the commutators at the shell radii. Consider a single negatively charged shell of radius $a$. The coefficient $P$ in $\mathbf{A}_{2}$ has a finite jump discontinuity at $r=a$, so we write

$$
P=\left(e^{2} \mu / \hbar\right)\left[Z_{<}^{\mathrm{eff}} \theta(a-r)+Z_{>}^{\text {eff }} \theta(r-a)\right]
$$

where $\theta(r-a)$ is the Heaviside function $\dagger$, and $Z_{<}^{\text {eff }} e$ and $Z_{>}^{\text {eff } e} e$ are the effective nuclear charges for $r<a$ and $r>a$, respectively. The term in $T$ involving,radial

\footnotetext{
* While $\left[\mathbf{A}_{1}, T\right]$ contains fourth derivatives with respect to Cartesian coordinates, it is easily shown to contain no derivatives higher than the third with respect to the radial coordinate $r$. As described in [20], not only are the piecewise Coulombic wave-functions and their first derivatives with respect to $r$ continuous at the shell radii, but also their second derivatives are continuous. The jump discontinuities in the third radial derivatives at the shell radii are finite, so that a Dirac $\delta$ function does not appear until fourth radial derivatives are taken. As such derivatives do not appear in $\left[\mathbf{A}_{1}, T\right]$, we may still take this commutator to equal zero.

$\dagger$ The function $\theta(x)$ equals zero for $x<0$ but unity for $x>0$, so that $d \theta / d$ equals $\delta(x)$, the Dirac delta function.
} 
derivatives is $-\left(\hbar^{2} / 2 \mu\right) r^{-2} \partial / \partial r\left(r^{2} \partial / \partial r\right)$, leading to

$$
\begin{aligned}
{\left[\mathbf{A}_{2}, T\right]=} & {[\text { terms in Eq. }(28)]+\left(e^{2} \hbar / 2\right)(\mathbf{r} / r) } \\
& \times\left(Z_{<}^{\text {eff }}-Z_{>}^{\text {eff }}\right)\left\{2 \delta(r-a)\left(r^{-1}+\partial / \partial r\right)+\delta^{\prime}(r-a)\right\}
\end{aligned}
$$

where $\delta(r-a)$ is the Dirac delta function and $\delta^{\prime}(r-a)$ is its derivative with respect to $r$. The terms in Eq. (31) which are like Eq. (28), but with the value of $P$ given by Eq. (30), are those which add to $\left[\mathbf{A}_{1}, V\right]$ to give zero. No terms involving $\delta(r-a)$ arise in $\left[\mathbf{A}_{1}, V\right]$, which contains $d V / d r$ but no higher derivatives of $V$, and none arise* in $\left[\mathbf{A}_{1}, T\right]$ or $\left[\mathbf{A}_{2}, V\right]$. Thus the finite jump discontinuity in $d V / d r$ at $r=a$ introduces a discontinuity in the definition of $\mathbf{A}_{2}$, using Eq. (30), leading to the result that $[\mathbf{A}, \mathscr{H}]$ does not vanish at the shell radius; hence $\mathbf{A}$ is not a constant of motion. It is interesting to note that the penetrating orbits obtained from the old quantum theory [21] for a two-region piecewise Coulombic field are segments of Kepler ellipses in each region. The change in the eccentricity and orientation of these segments, and hence in the value of $\mathbf{A}$, in passing from one region to the other results in an advancing perihelion and an overall rosette orbit. As discussed by Born [22], this description may be extended to the $n$-region piecewise Coulombic field.

In summary, it is concluded that matrix elements of the Runge-Lenz vector $\mathbf{A}$ provide a useful description of polar wave-functions such as those linear combinations of atomic orbitals known as hybrid orbitals. Specifically, the matrix elements of $\mathbf{A}$ help establish the correspondence between wave-mechanical and classical descriptions. The properties of $\mathbf{A}$ are described for hybrid orbitals constructed from general central-field functions for which $\mathbf{A}$ is not diagonal in the principal quantum number $n$. As a consequence, these hybrid functions are characterized by greater spreads $\sigma(\mathbf{A})$ than are their hydrogenic counterparts. Analysis of the properties of a piecewise Coulombic field shows that the noncommutation of $\mathbf{A}$ and $\mathscr{H}$ is associated with a discontinuity in the radial derivative of the potential energy. ${ }^{\dagger}$

\section{Acknowledgment}

The author wishes to thank Professor S. M. Blinder and Dr. D. F. Heller for valuable discussions, and the University of California, Berkeley for its hospitality during the 1974-5 academic year. He also wishes to thank Professor C. Wulfman for helpful discussions of group theory.

\section{Bibliography}

[1] See the reviews by M. Bander and C. Itzykson, Rev. Mod. Phys. 38, 330, 346 (1966); H. V. McIntosh, Group Theory and its Applications, Vol. II, E. M. Loebl, Ed. (Academic Press, New York, 1971), p. 75; C. Wulfman, ibid., p. 145. The invariance with respect to $O_{4}$ was first pointed out in V. Fock, Z Physik 98, 145 (1935) and V. Bargmann, Z. Physik 99, 576 (1936).

* See footnote on p. 807.

$\dagger$ The piecewise Coulombic example suggests a choice of $P$ for smooth central fields not as $Z e^{2} \mu / \hbar$, where $Z e$ is the true nuclear charge, but instead as $r^{2}(d V / d r) \mu / \hbar$, which is the choice in Eq. (27), making $[\mathbf{A}, H]=0$ within each piecewise Coulombic region. However, it is unclear whether the resulting definition of $\mathbf{A}$ has any physical significance. 
[2] M. J. Englefield, Group Theory and the Coulomb Problem (Wiley-Interscience, New York, 1972).

[3] W. Pauli, Z. Physik 36, 336 (1926) [for English translation see B. L. van der Waerden, Sources of Quantum Mechanics (North-Holland Publishing Co., Amsterdam, 1967), p. 387.]

[4] C. Runge, Vector Analysis (Dutton, New York, 1919), p. 79; W. Lenz, Z. Physik 24, 197 (1924).

[5] J. S. Alper and O. Sinanoglǔ, Phys. Rev. 177, 77 (1969).

[6] J. S. Alper, Phys. Rev, 177, 86 (1969).

[7] E. Chacon, M. Moshinsky, O. Novaro, and C. Wulfman, Phys. Rev. A3, 166 (1971).

[8] C. Wulfman, Chem. Phys. Lett. 23, 370 (1973).

[9] O. Sinanoglŭ and D. R. Herrick, Chem. Phys. Lett. 31, 373 (1975).

[10] D. R. Herrick and O. Sinanoglŭ, Phys. Rev. A11, 97 (1975).

[11] O. Sinanoglŭ and D. R. Herrick, J. Chem. Phys. 62, 886 (1975).

[12] D. R. Herrick, J. Math. Phys. 16, 1047 (1975).

[13] C. Wulfman and S. Kumei, Chem. Phys. Lett. 23, 367 (1973).

[14] C. A. Coulson and A. Joseph, Int. J. Quantum Chem. 1, 337 (1967).

[15] H. Bacry, R. Ruegg, and J. Souriau, Commun. Math. Phys. 3, 323 (1966).

[16] N. Mukunda, Phys. Rev. 155, 1383 (1967).

[17] U. Fano and G. Racah, Irreducible Tensorial Sets (Academic Press, New York, 1959).

[18] J. S. Griffith, The Irreducible Tensor Method for Molecular Symmetry Groups (Prentice-Hall, Englewood Cliffs, N.J., 1962).

[19] E. J. McGuire, Phys. Rev. 161, 51 (1967); 175, 20 (1968); also see Phys. Rev. A3, 267 (1971) for applications to inelastic electron and proton scattering.

[20] F. M. Chapman, Jr. and L. L. Lohr, Jr., J. Amer Chem. Soc. 96, 4731 (1974).

[21] E. Schrödinger, Z. Physik 4, 347 (1921); also see H. E. White, Introduction to Atomic Spectra (McGraw-Hill, New York, 1934), p. 106 and references cited therein.

[22] M. Born, The Mechanics of the Atom (G. Bell and Sons, Ltd., London, 1927), p. 169.

Received May 10, 1975

Revised September 23, 1975 